\title{
Intermittent fluid connectivity during two-phase flow in a heterogeneous carbonate rock
}

\author{
Catherine Spurin $\odot,{ }^{1, *}$ Tom Bultreys, ${ }^{1,2}$ Branko Bijeljic, ${ }^{1}$ Martin J. Blunt, ${ }^{1}$ and Samuel Krevor ${ }^{1}$ \\ ${ }^{1}$ Department of Earth Science \& Engineering, Imperial College London SW7 2BP, United Kingdom \\ ${ }^{2}$ Department of Geology, Ghent University, Krijgslaan 281 S8, 9000 Ghent, Belgium
}

(Received 3 July 2019; published 8 October 2019)

\begin{abstract}
Subsurface fluid flow is ubiquitous in nature, and understanding the interaction of multiple fluids as they flow within a porous medium is central to many geological, environmental, and industrial processes. It is assumed that the flow pathways of each phase are invariant when modeling subsurface flow using Darcy's law extended to multiphase flow, a condition that is assumed to be valid during steady-state flow. However, it has been observed that intermittent flow pathways exist at steady state even at the low capillary numbers typically encountered in the subsurface. Little is known about the pore structure controls or the impact of intermittency on continuum scale flow properties. Here we investigate the impact of intermittent pathways on the connectivity of the fluids for a carbonate rock. Using laboratory-based micro computed tomography imaging we observe that intermittent pathway flow occurs in intermediate-sized pores due to the competition between both flowing fluids. This competition moves to smaller pores when the flow rate of the nonwetting phase increases. Intermittency occurs in poorly connected pores or in regions where the nonwetting phase itself is poorly connected. Intermittent pathways lead to the interrupted transport of the fluids; this means they are important in determining continuum scale flow properties, such as relative permeability. The impact of intermittency on flow properties is significant because it occurs at key locations, whereby the nonwetting phase is otherwise disconnected.
\end{abstract}

DOI: 10.1103/PhysRevE.100.043103

\section{INTRODUCTION}

Understanding multiphase fluid flow in porous media is important due to its presence in a broad range of applications including carbon sequestration, hydrocarbon recovery and aquifer contaminant containment. Subsurface reservoirs are typically modelled using relative permeability and capillary pressure as the constitutive properties [1-3]. However, these properties are used in combination with an extension of Darcy's law to multiphase flow under the justification that, for a given saturation, the interfaces between fluid phases will remain fixed [4-6]. Steady state is achieved once the saturation of the fluid phases is statistically invariant in time [7-9]. An equilibrium pore-scale distribution of fluids is assumed in the continuum description of subsurface reservoirs. Thus the flow properties required when modeling reservoirs are often derived from observations made under steady-state conditions with rock samples in the laboratory. It is assumed that, at steady state, any disconnected regions are trapped, and only a smooth, slow evolution of saturation and fluid configuration in space and time is possible [10,11]. The flow of each fluid phase is assumed to be independent of the other fluids phases present [5].

However, interface dynamics at steady state have been observed, from the discrete movement of nonwetting phase clusters, termed ganglion dynamics $[12,13]$ to the periodic disconnection and subsequent reconnection of the nonwetting phase, termed dynamic connectivity or intermittent pathway flow [4,14-19]. Recently, we showed that the generation of

\footnotetext{
*cls13@ic.ac.uk
}

these deviations from connected pathway flow are predominantly controlled by the ratio of viscous to capillary forces (classified by the dimensionless capillary number, $\mathrm{Ca}$ ) and the viscosity ratio [19]. Intermittency is of particular relevance to practical applications of subsurface flow due to its occurrence at relatively low flow rates, where capillary forces are dominant.

It is not well understood what impact intermittent pathway flow has on larger scale flow properties such as the relative permeability or capillary pressure. The continuous rearrangement of interfaces will influence the energy dissipation in the system and will, therefore, affect the transmission of fluids. Varying fluid connectivity caused by intermittent pathways cannot be ignored for accurate pore-scale flow simulation or for predictive pore-network models of continuum scale properties, with fluid topology and phase connectivity considered important parameters missing from current multiphase flow models [4].

Further research is required to explore how local pore structural characteristics and fluid occupancy lead to intermittency in a given location. Pore geometry is known to control the snap-off events [20] that will cause the disconnection of intermittent pathways. Reconnection events will be influenced by local fluid occupancy as reinvasion is more favorable through cooperative pore filling of the wetting phase [21]. Understanding the role of these displacement dynamics during steady-state intermittent pathways will provide insight into the location, size, and critical capillary pressures of the intermittent pathways and thus provide information on how these events control the transport of the fluids and influence the relative permeability and capillary pressure $[21,22]$. 
TABLE I. Fluid parameters. Density values for decane and hexadecane from VWR International. Viscosity and interfacial tension for decane from Ref. [15] and for hexadecane from Refs. [25] and [26]. Remaining values from PubChem, open chemistry database.

\begin{tabular}{lccc}
\hline \hline Fluid & $\begin{array}{c}\text { Viscosity } \mu \\
(\mathrm{Pa} \mathrm{s})\end{array}$ & $\begin{array}{c}\text { Density } \rho \\
\left(\mathrm{kg} / \mathrm{m}^{3}\right)\end{array}$ & $\begin{array}{c}\text { Interfacial tension } \\
\text { with brine } \sigma(\mathrm{N} / \mathrm{m})\end{array}$ \\
\hline Nitrogen & $2.08 \times 10^{-5}$ & 110 & $47 \times 10^{-3}$ \\
Decane & $83.8 \times 10^{-5}$ & 730 & $64 \times 10^{-3}$ \\
Hexadecane & $34.5 \times 10^{-4}$ & 773 & $53 \times 10^{-3}$ \\
Brine & $82.0 \times 10^{-5}$ & 1263 & $\mathrm{~N} / \mathrm{A}$ \\
\hline \hline
\end{tabular}

In recent work, we explored the controlling parameters for intermittency and found that intermittent pathway flow is possible at the low capillary numbers typical of subsurface flow, especially when the viscosity of the nonwetting phase is much lower than that of the wetting phase [19]. In this work, we explore the pore-scale physics controlling the occurrence and location of intermittent pathways and its link to fluid connectivity for steady-state two-phase flow. We assess the location of intermittent events with respect to the local fluid distribution and pore structure and determine their impact on fluid connectivity for the fluid pairings and capillary numbers described by Spurin et al. [19].

\section{METHODS}

\section{A. Materials}

An Estaillades carbonate cylindrical rock sample, $6 \mathrm{~mm}$ in diameter and $21 \mathrm{~mm}$ in length, was studied. It was chosen for its pore space complexity as a bioclastic carbonate rock. Previous studies using homogeneous sandstones explored intermittency for both nitrogen-brine [14] and decane-brine flows [15]. The more complicated rock structure used in this study allowed the role of pore geometry to be studied. The sample was cleaned with methanol in a Soxhlet extractor for $24 \mathrm{~h}$ and dried in a vacuum oven overnight. The sample was water-wetting. Estaillades is typically more than $97 \%$ calcite, with the remaining material being quartz [23]. The helium porosity measured for a similar Estaillades carbonate sample was $29 \%$, although some of this porosity was below the resolution of the micro computed tomography (micro-CT) scanner [24].

The wetting phase, brine, was deionized water doped with $25 \%$ wt. potassium iodide to achieve a clear contrast between phases in the x-ray scans. The nonwetting phases used were nitrogen, decane, or hexadecane. The properties of all fluids used are given in Table I.

\section{B. Experimental procedure}

Two-phase, steady-state coinjection experiments were performed in the apparatus shown in Fig. 1 as described by Spurin et al. [19] but reported here for completeness. There are four sets of experiments described in this work with the nonwetting phase and/or total flow rate of both fluids varied between experiments. During an experiment, the fractional flow $\left(f_{w}\right)$ was decreased in a series of steps; these were therefore drainage experiments. The fractional flow is defined

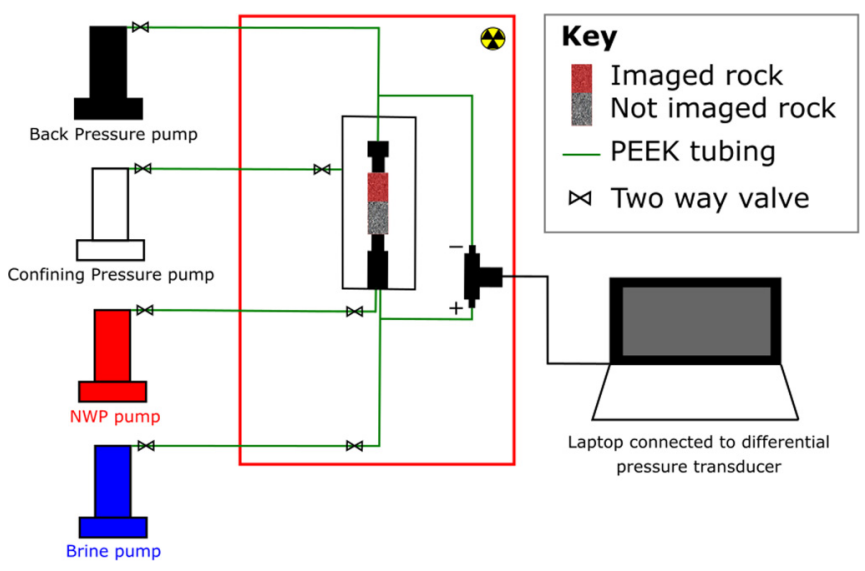

FIG. 1. Schematic of the flow loop used for the experiments. Only the top part of the sample was imaged.

as the fraction of the total (oil and brine) flow rate constituted by the brine. The four sets of experiments are as follows:

(1) Nitrogen-brine injection at $0.8 \mathrm{ml} / \mathrm{min}$ for $f_{w}=1$, $0.85,0.7,0.5,0.25,0.03,0$. The observations from this experiment are referred to as the high-Ca nitrogen observations. The capillary number ranged from $1.5-9.0 \times 10^{-6}$.

(2) Nitrogen-brine injection at $0.1 \mathrm{ml} / \mathrm{min}$ for $f_{w}=1$, $0.85,0.7,0.5,0.25,0.03,0$. These are referred to as the low-Ca nitrogen observations. The capillary number ranged from $0.2-1.1 \times 10^{-6}$.

(3) Decane-brine injection at $0.02 \mathrm{ml} / \min$ for $f_{w}=1$, $0.85,0.7,0.5,0.25,0$. These are referred to as the decane observations. The capillary number ranged from 2.1 to $2.2 \times$ $10^{-6}$.

(4) Hexadecane-brine injection at $0.02 \mathrm{ml} / \min$ for $f_{w}=1$, $0.85,0.7,0.5,0.25,0$. These are referred to as the hexadecane observations. The capillary number ranged from 2.2 to $4.5 \times$ $10^{-6}$.

The nitrogen experiments were conducted at $10 \mathrm{MPa}$ to minimize the compressibility of the nitrogen gas in the pore space, whereas decane and hexadecane experiments were conducted at ambient pressures.

The experimental procedure, once the carbon fiber flow cell (Airbourne Composites) had been assembled and placed into the micro-CT scanner (Zeiss Versa), was as follows:

(1) A confining pressure of $2 \mathrm{MPa}$ was applied to the sample within the flow cell.

(2) Only the top half of the sample was imaged. This required three separate circular scans with approximately $20 \%$ overlap, so that the images could be stitched together during image processing.

(3) The first set of scans were taken with only air in the pore space; these are the air-saturated scans.

(4) Air was removed from the sample by flushing with brine at $10-\mathrm{MPa}$ back pressure, with the confining pressure always kept at an additional $2 \mathrm{MPa}$ over the pore pressure.

(5) Scans were taken to confirm that the sample was fully saturated with brine; these are the brine-saturated scans.

(6) The nonwetting phase was injected simultaneously with the brine, with constant back pressure. The fractional 
(a)

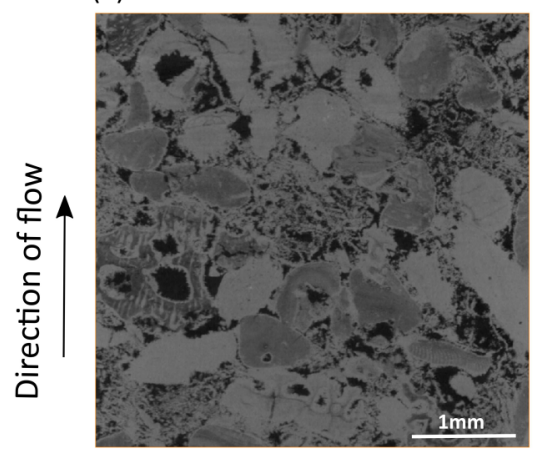

(c)

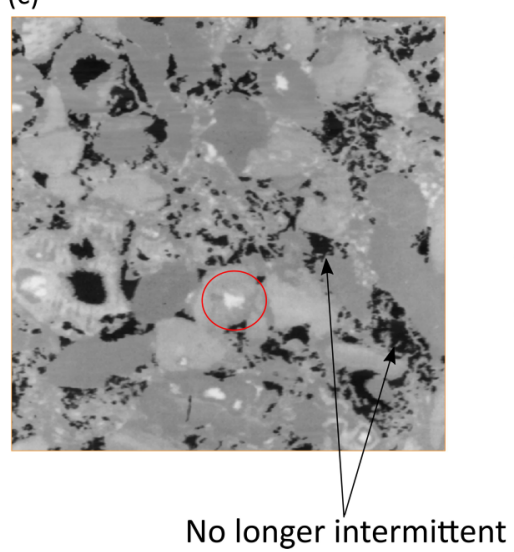

(b)

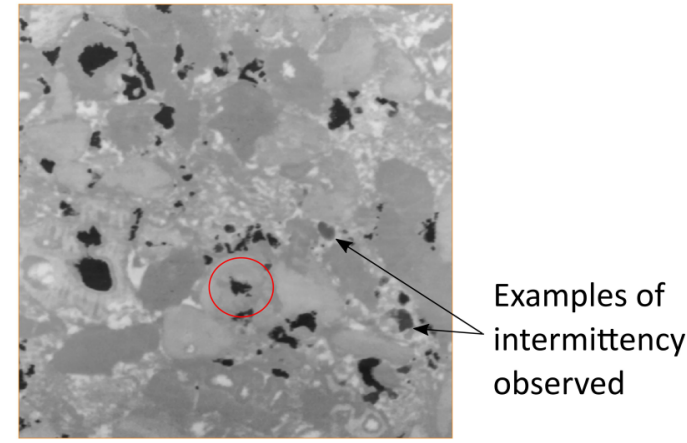

(d)

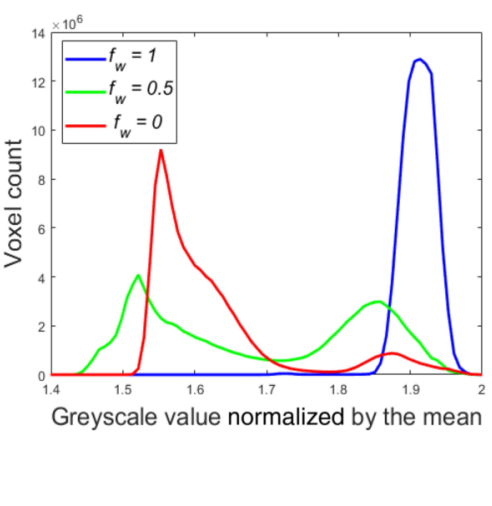

FIG. 2. (a) Grayscale data for the air-saturated scan, (b) grayscale data for the $f_{w}=0.5$ high-Ca experiment, (c) grayscale data for $f_{w}=$ 0 high-Ca experiment, and (d) histogram for the grayscale values in the pore space for (b) and (c) and the brine-saturated scan. Red circles highlight the rearrangement of fluid between scans.

flow $f_{w}$ was decreased while the total volumetric flow rate was kept constant during an experiment.

(7) Scans were acquired after steady state had been reached. This was determined to be the case when the differential pressure measured across the sample was observed to be stable and constant $[7,8,15]$. Injection of the fluids continued during a scan.

(8) The fractional flow was changed and the previous step was repeated.

At the end of an experiment, the sample was flushed with deionized water at $1 \mathrm{ml} / \mathrm{min}$ for more than $5 \mathrm{~h}$ and dried in a vacuum oven for $12 \mathrm{~h}$ at $95{ }^{\circ} \mathrm{C}$. The $\mathrm{x}$-ray source was set at a voltage of $80 \mathrm{kV}$ and a target power of $7 \mathrm{~W}$. A scan lasted approximately $40 \mathrm{~min}$, with 1601 projections taken to ensure good image quality.

\section{Image processing}

The nitrogen-brine scans had a voxel size of $3.9 \mu \mathrm{m}$ and the decane-brine and hexadecane-brine scans had a voxel size of $3.6 \mu \mathrm{m}$. A subvolume of $1000^{3}$ voxels was analyzed for all scans. The scans taken at each fractional flow were registered to the air-saturated scan before the subvolume was extracted. All subvolumes were filtered with a nonlocal means filter. The air-saturated scan, Fig. 2(a), provided a high-spatialresolution image of the rock prior to the injection of the fluids.

The pore space was segmented from the rock grains using the watershed segmentation algorithm [27]. The pores were identified by applying a maximal inscribed spheres network extraction algorithm on the segmented air-saturated image $[28,29]$. The network extraction identifies pores (wide regions) and throats (a restriction bounding two pores). The inscribed spheres for the pores were overlain on the segmented fractional flow images to determine the relationship between pore size and phase occupancy. The maximum inscribed spheres each have an associated number of nearestneighboring spheres, which are the number of pores directly connected to that pore by a region classified as a throat. The number of these directly neighboring pores is the coordination number for that pore.

A scan lasted approximately $40 \mathrm{~min}$ to collect enough $\mathrm{x}$-ray counts for the images to be reconstructed with minimal artifacts. Intermittent pathways were then inferred from the grayscale value recorded in the pore space; they could not be resolved directly because the timescales of interface rearrangement is well below the time resolution of a scan, perhaps on the same order of timescale as a Haines jump, which is milliseconds [30]. The grayscale value recorded for a voxel depends on the average atomic number of the atoms and the density within the voxel. If there was no movement during a scan, then only two grayscale values would be identifiable in the pore space: one for the nonwetting phase and one for brine. If both brine and the nonwetting phase occupy a pore at some point during a scan, then the resulting image will have a grayscale value between the two phases, dependent on the amount of time each phase was occupying 
(a)

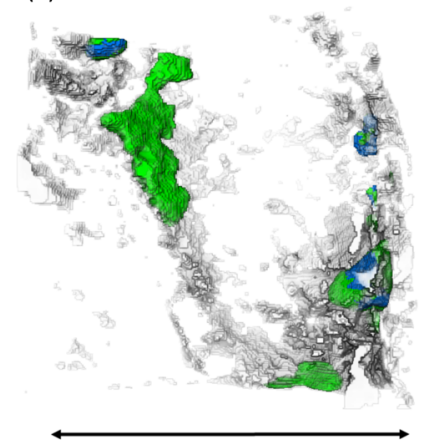

$0.8 \mathrm{~mm}$ (b)

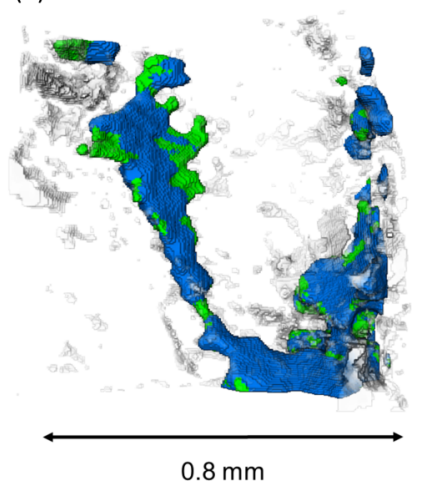

FIG. 3. Intermittency classification in three dimensions for a subvolume within the sample: (a) $f_{w}=0.7$ high-Ca nitrogen experiment and (b) $f_{w}=0.25$ high-Ca nitrogen experiment. Green is a region of intermittent flow behavior and blue is stable nitrogen. The pore space is overlain. The average flow direction is top to bottom.

that pore, resulting in an intermediate grayscale value. This intermediate grayscale was segmented as a region with intermittent pathway flow [19]. Note that no axial dependency was observed for the size of a region with intermittent pathway flow, providing support that the boundaries are not influencing the results of this work.

For the brine-saturated scan, only brine was present in the pore space; this is represented by a single peak in the grayscale values for the pore space [the blue line in Fig. 2(d)]. When the nonwetting phase was introduced, for example, in the $f_{w}$ $=0.5$ high-Ca nitrogen scan, a second peak is observed in the grayscale values within the pore space [the green line in Fig. 2(d)]. However, manual inspection of the scan in Fig. 2(b) shows there is a third, intermediate grayscale value between the dark nonwetting phase and the bright brine in the pore space. This intermediate grayscale was not present for the $f_{w}$ $=0$ high-Ca scan in Fig. 2(c).

Intermittency is caused by periodic occupancy of both phases and so both phases would be present in the same part of the pore space during a scan. This means that the intermediate grayscale value can be interpreted as the intermittent presence of brine and the nonwetting phase. As the grayscale value of regions with intermittent flow behavior depends on the amount of time each phase spends in the pore during the scan, these regions do not create an observable peak in the pore space histogram. Further information on the processing of the images is detailed by Spurin et al. [19].

\section{RESULTS AND DISCUSSION}

\section{A. Role of intermittency in fluid connectivity}

The role of intermittency in the distribution of phases is important for determining the connectivity of both fluids and thus their relative permeability. Figure 3 qualitatively shows the importance of intermittency in connecting otherwise-isolated regions of the nonwetting phase, with intermittency providing a connection between two stable regions for Fig. 3(b). Figure 3 also shows how areas exhibiting intermittent flow behavior can become stable as $f_{w}$ decreases.

A number of trends can be identified in the observations. More intermittency was observed in the high-Ca nitrogen experiments compared to the low-Ca nitrogen experiments, and more intermittency was observed in the nitrogen-brine experiments than the decane-brine experiments, as shown in Fig. 4(a). No intermittency was observed in the hexadecane experiments. This has been reported in Spurin et al. [19] and is primarily caused by the viscosity ratio of the fluids. The fraction of the pore space where intermittency occurs increases from $f_{w}=0.85$ to $f_{w}=0.7$ and then decreases from $f_{w}=0.5$ for the nitrogen experiments. A similar peak is observed for the decane experiments and also at higher capillary numbers for decane-brine experiments conducted in a water-wet Bentheimer sandstone sample [15]. For $f_{w}>0.5$, the nonwetting phase flow rate is small in comparison to the total flow rate; this means that the saturation of the nonwetting phase is lower, which would encourage more transport via intermittency. Interestingly, in an oil-wet Bentheimer sandstone sample, a peak was observed at the opposite end of the fractional flow range at $f_{w}=0.1$ [31]. Here the water was the nonwetting phase and hence again intermittency was most prevalent when the nonwetting phase was poorly connected.

Intermittent flow pathways are crucial in evaluating the connectivity of the nonwetting phase from micro-CT images because they provide flow pathways for nonwetting phase clusters that appear trapped when intermittency is not considered. The number of discrete ganglia was calculated for the volumes classified as stable nonwetting phase and then recalculated with the volumes classified as intermittent pathways also included as nonwetting phase. The fractional change in the number of ganglia through the inclusion of the intermittent phase is shown in Fig. 4(b). We see a significant change in the number of ganglia-up to $55 \%$ for the high$\mathrm{Ca}$ nitrogen experiment. For all but the high-Ca nitrogen case, the magnitude of the reduction mirrors the fraction of intermittent phase shown in Fig. 4(a). These results indicate the importance of intermittent fluid dynamics in connecting areas of stable fluid occupancy.

The observations show intermittency playing a key role in connectivity across a range of conditions. This means that the inclusion of intermittent pathways cannot be ignored from the assessment of fluid transmissibility; it influences the connectivity of the fluids and thus impacts relative permeability estimates for both phases. The scan with the highest percentage of pore space identified as intermittent was the $f_{w}=0.7$ high-Ca nitrogen scan, with $21 \%$ of the segmented pore space classified as intermittent flow. It can be seen from Fig. 4(b) that the inclusion of intermittent regions for this scan greatly reduces the number of distinct nonwetting phase ganglia observed; that is, the connectivity of the nonwetting phase is greatly increased with intermittency. However, even for the scans with less intermittency (on the order of $5 \%$ of the segmented pore space) there was a significant difference between the number of nonwetting phase ganglia observed with and without the inclusion of the intermittent phase for the high-Ca nitrogen experiments. This shows that intermittent flow pathways occur at key junctions for the nonwetting phase and influence the connectivity of the nonwetting phase significantly. For $f_{w}<0.5$, for both the low-Ca nitrogen and the decane observations, the volumes defined as intermittent 


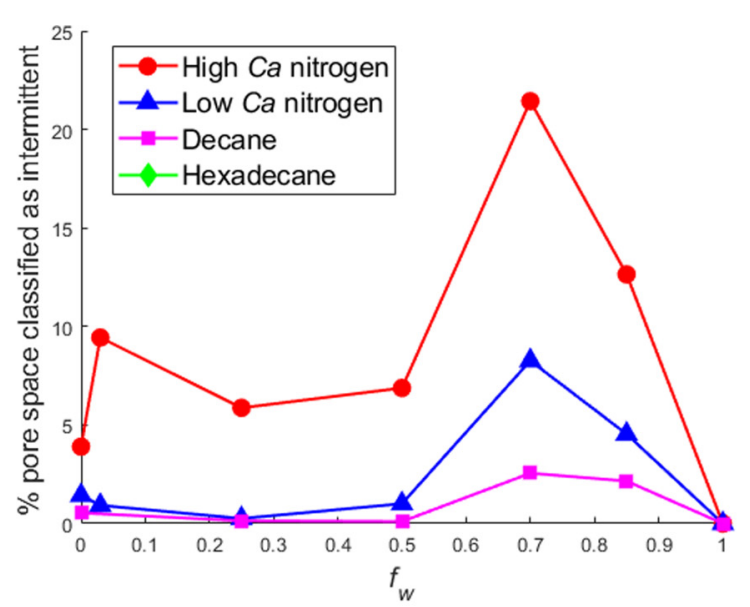

(a)

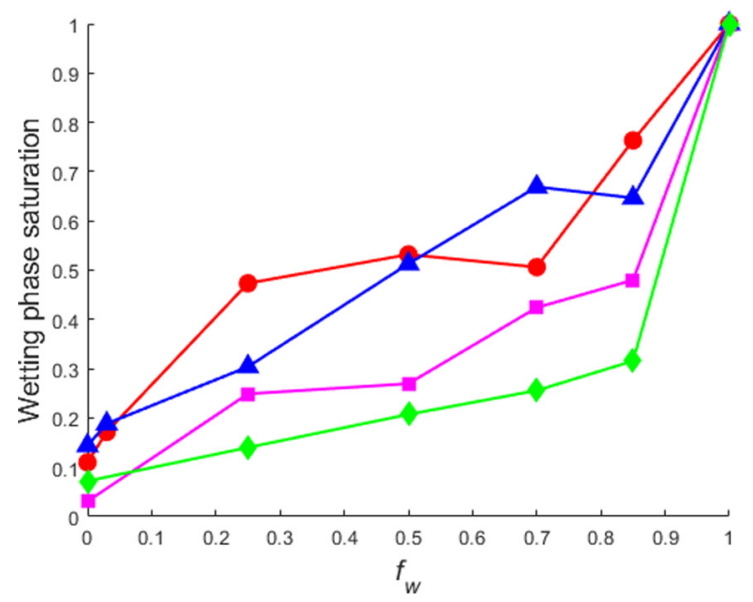

(c)

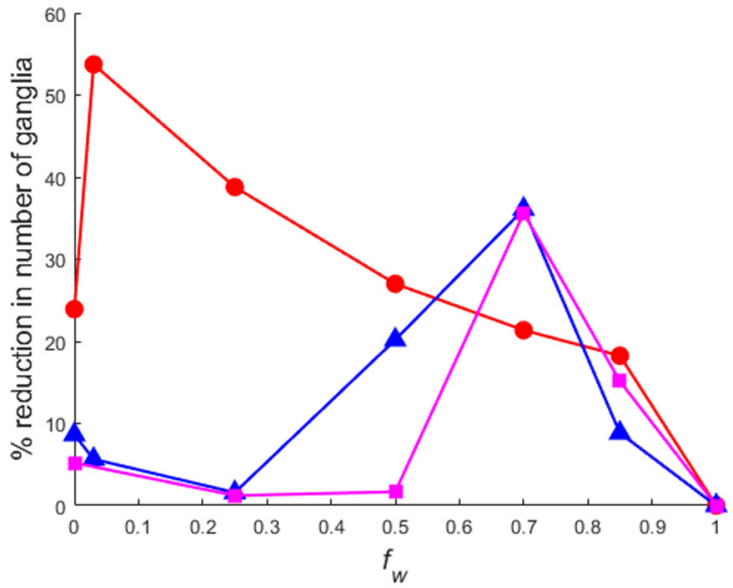

(b)

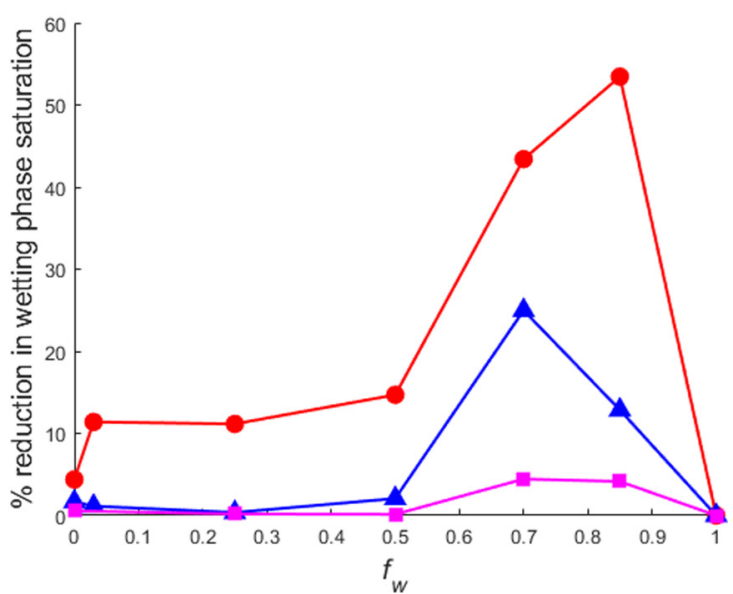

(d)

FIG. 4. The role of intermittency on the connectivity of the nonwetting phase for all experiments: (a) percentage volume of the pore space identified as intermittent, (b) fractional reduction in the number of ganglia through the inclusion of intermittent flow pathways as stable nonwetting phase, (c) brine saturation, and (d) change in the brine saturation through the inclusion of intermittent flow pathways as stable nonwetting phase.

constitute a small percentage of the total pore space. The change in the number of ganglia through the inclusion of intermittent regions as stable nonwetting phase is much lower for these images. However, the role of intermittent pathways is still important at higher $f_{w}$ for the low-Ca nitrogen and the decane observations.

\section{B. Impact of heterogeneity in generating intermittency}

For the decane experiments, only a small percentage of the pore space was identified as intermittent [see Fig. 4(a)], but no intermittency at all was seen at similar capillary numbers for decane-brine experiments conducted in Bentheimer sandstone [15]. This suggests that a more variable pore geometry in Estaillades carbonate encourages intermittent pathway flow.

The impact of the heterogeneity of Estaillades was also assessed by conducting a repeat observation for the lowCa nitrogen experiment and analyzing the same subvolume shown in Fig. 4 (denoted as subvolume 1 in Fig. 5) and by comparing this with observations in a different $1000^{3}$ voxels subvolume within the sample (subvolume 2 in Fig. 5). This allows variations in intermittent pathway flow behavior caused by the variation in the distribution of the fluids (which occur in the repeat experiment by slight variations in the initial displacement) to be compared with variations in intermittent pathway flow within a sample caused by a heterogeneous pore size distribution.

The saturation [Fig. 5(c)] and change in number of ganglia [Fig. 5(b)] for the same subvolume are similar between the repeat experiment and the original experiment. However, the percentage volume of the pore space that was identified as intermittent was much lower for the repeat experiment when $f_{w}>0.5$. The same peak at $f_{w}=0.7$ is still observable in Fig. 5(a).

Experiments by Andrew et al. [24] exploring the trapping of supercritical $\mathrm{CO}_{2}$ by brine in Estaillades and other limestones showed that pore occupancy changes when experiments are repeated. However, the trapped ganglion size distributions and average saturation were comparable between experiments, even with this change in pore occupancy. This implies that although slight changes in the initial conditions cause variations in the subsequent pore displacement events, the nonwetting phase still occupies the largest pores, leading to comparable saturation, while the trapping mechanisms are 


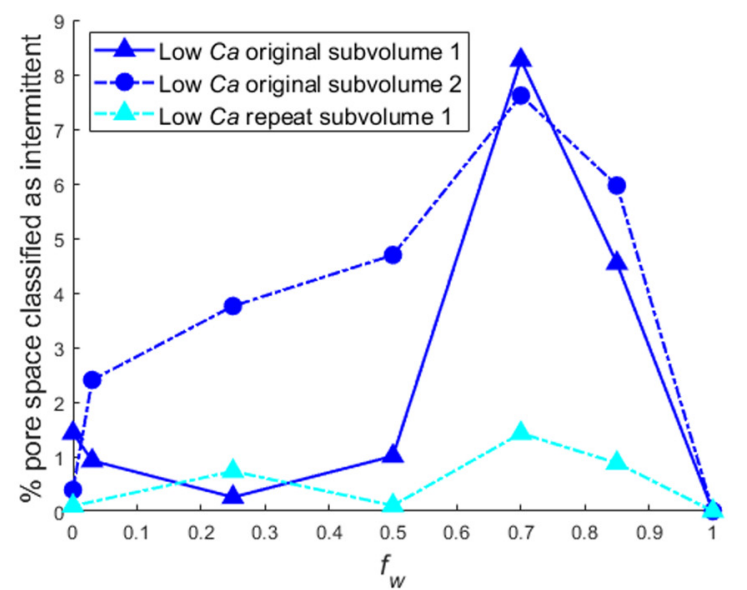

(a)

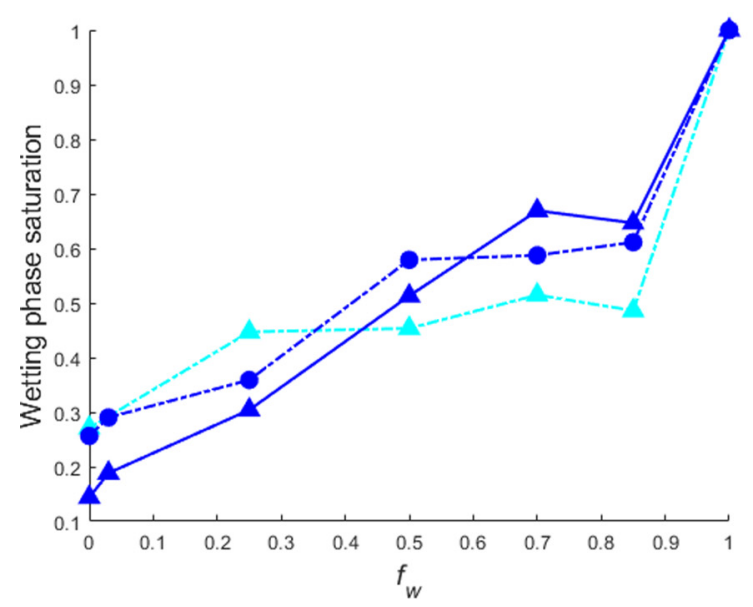

(c)

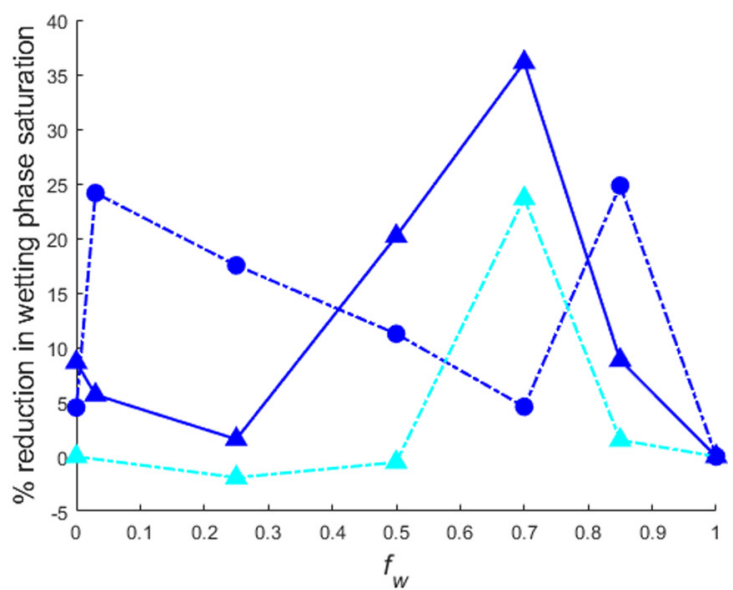

(b)

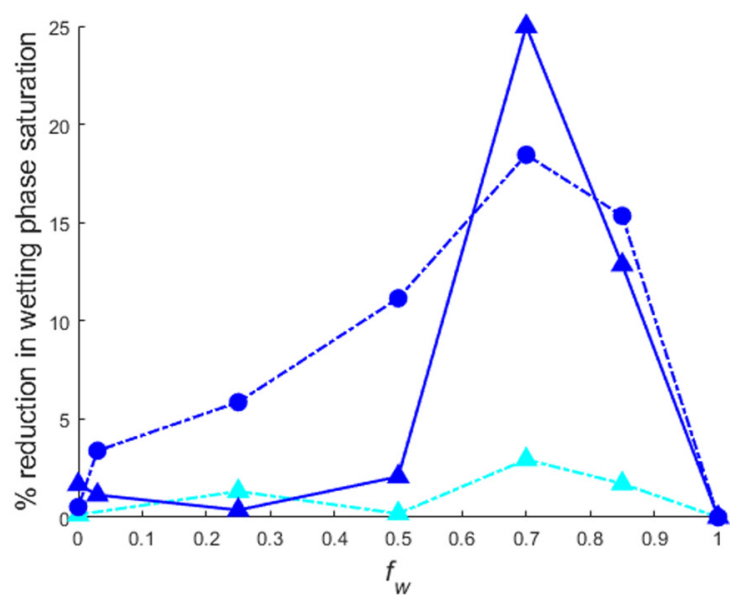

(d)

FIG. 5. The role of intermittency in the connectivity of the nonwetting phase for a different subvolume and for a repeat experiment for the low-Ca nitrogen experiments: (a) Percentage volume of the pore space identified as intermittent, (b) fractional reduction in the number of ganglia through the inclusion of intermittent flow pathways as stable nonwetting phase, (c) brine saturation, and (d) change in the brine saturation through the inclusion of intermittent flow pathways as stable nonwetting phase.

similar, leading to comparable ganglion sizes [32]. This offers an explanation to why the amount of intermittency is variable for the same region for the repeat experiment; the intermittency is not just controlled by the local conditions, but by the total network of fluid phases distributed throughout the pore space. This means the amount of intermittent pathway flow observed will be highly variable when considering a small subvolume in a much larger domain.

For subvolume 2, the saturation is consistent with subvolume 1 [Fig. 5(c)]. The change in the number of ganglia through the inclusion of areas identified as intermittent pathways as nonwetting phase is significantly higher for subvolume 2 [Fig. 5(b)]. The peak of intermittency identified at $f_{w}=0.7$ is also observable in subvolume 2 . The decrease in intermittent pathways identified once the nitrogen flow rate dominates over the brine flow rate is much less pronounced, although it still exists. This highlights the importance of the pore geometry in controlling not only the amount of periodic pathway flow but also the impact of this flow on the connectivity of the nonwetting phase. The representative elementary volume (REV) for intermittency has not been characterized in this work, but it is evident that we are not at the scale of an REV in these observations as the variation between the subvolumes is not negligible, which would be expected at steady state [33].

It must be noted that the sample was removed from its sleeve between the repeat low-Ca experiment and the other experiments. This means that, although the subvolume identified is the same, the outside boundary conditions have changed, as outer grains are lost during the removal of the sleeve.

\section{Location and size of intermittent pathways}

Figure 6 shows that nitrogen or decane, as the nonwetting phase, occupied the largest pores. The brine, as the wetting phase, occupied the smaller pores (and the microporosity, which is not explored as it was never occupied by the nonwetting phase). The intermittent phase occupies the lower end of pore sizes typically occupied by the nonwetting phase, as shown most clearly in Fig. 6(a), where the most intermittency was observed. The nonwetting phase continuously occupies the larger pores with lower entry capillary pressures but intermittency occurs in the intermediate-sized pores where brine and the nonwetting phase are competing. In these 


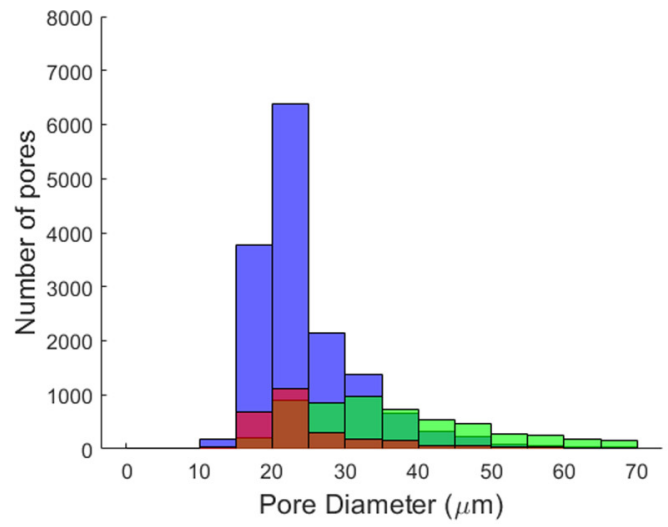

(a)

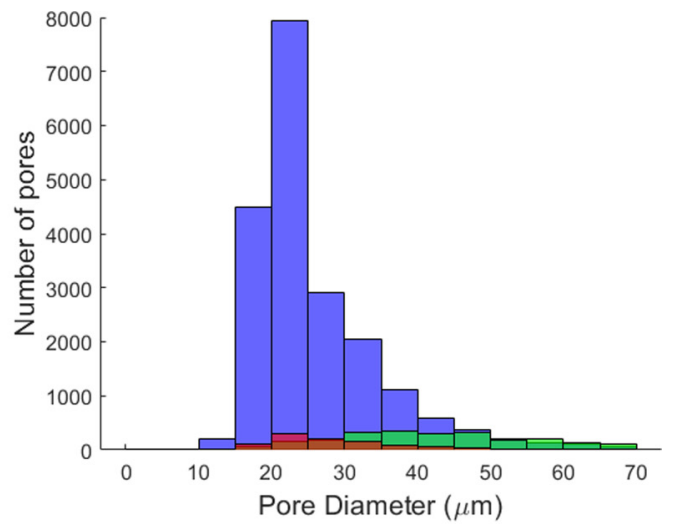

(b)

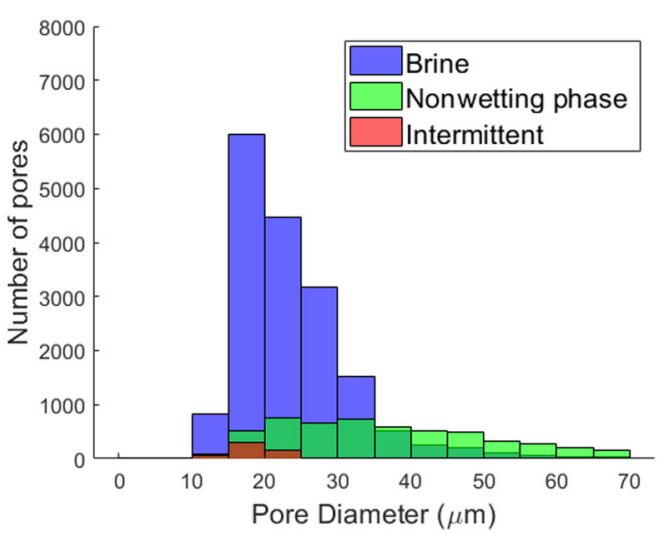

(c)

FIG. 6. Pore occupancy for all experiments for the $f_{w}=0.7 \mathrm{scan}$, where the most intermittency was identified. Pore diameter was classified in bins of $5 \mu \mathrm{m}$ : (a) high-Ca nitrogen, (b) low-Ca nitrogen, and (c) decane.

locations the local pressure difference between the two phases is close to the capillary entry pressure. This means that the nonwetting phase can invade, but the invasion is sensitive to pressure perturbations and thus susceptible to oscillations in pore occupancy.

As the nonwetting phase capillary number increases (and $f_{w}$ decreases), the average pore size occupied by the nonwetting phase decreases, as it is able to invade smaller pores (Fig. 7). The average pore size for intermittency also decreases

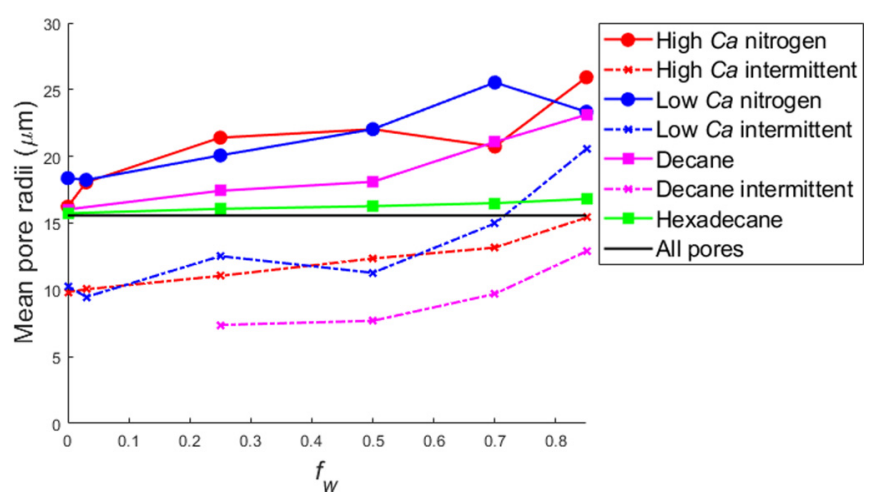

FIG. 7. Mean pore size for the nonwetting phase filled pores and intermittent pores for all experiments. The black line defines the mean pore size for all pores within the subvolume. with decreasing $f_{w}$, as shown in Fig. 7. This supports the hypothesis that a competition between phases occurs when the local pressure is close to the entry pressure for the nonwetting phase. As the flow of the nonwetting phase increases, so does its contribution to the capillary number [19]. Increasing the energy of the nonwetting phase injected into the system allows it to occupy smaller pores intermittently.

The mean pore size for pores with intermittency is below the mean pore size for all observations, except for $f_{w}=$ 0.85 low-Ca nitrogen (Fig. 7). For $f_{w}=0$ decane, although intermittency was identified in some of the pore space, it was solely in regions defined as throats in the network extraction. This emphasizes the stability of the interfaces imaged in the decane experiments because the inscribed spheres for the pores are less prone to the incorrect segmentation of the partial volume effects as intermittency. The mean pore size occupied by both the nonwetting phase and intermittency is lower for the decane experiments than the nitrogen experiments for a given fractional flow. This is the expected result of a higher viscosity ratio for decane-brine and is also reflected as a higher saturation.

Although the experiments were macroscopically drainage displacements, where the nonwetting phase displaced brine, intermittent behavior will consist of both nonwetting phase displacing the brine and vice versa. This means that the local capillary entry pressure is not solely controlled by pore size; the filling mechanism is also important for determining 


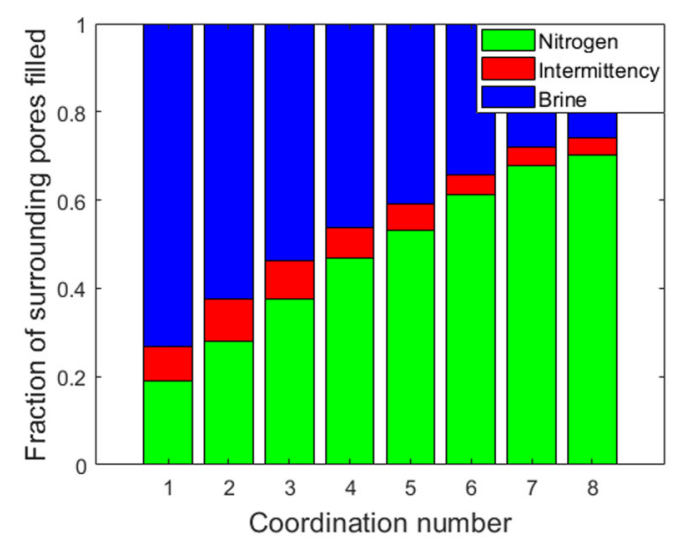

(a)

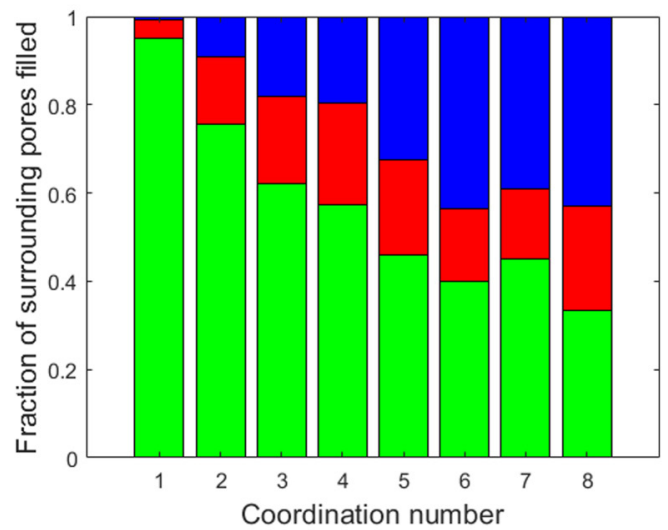

(b)

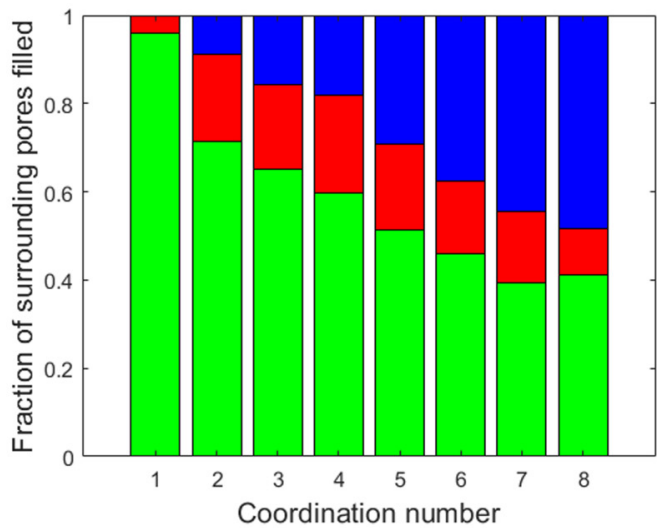

(c)

FIG. 8. Coordination number against pore occupancy of the nearest-neighbor pores: (a) All pores for all nitrogen observations, (b) intermittent pores for the low-Ca nitrogen observations and, (c) intermittent pores for the high-Ca nitrogen observations.

entry pressure, as it controls the meniscus of the interface between phases [21]. If the nonwetting phase invades a pore, then the brine is much more likely to reenter if it can do so cooperatively. This will be the case where most of the neighboring pores are also filled with brine.

To test this, the intermittent pores were identified along with the phase occupancy of the pores directly connected to that pore. This has been done for the nitrogen experiments, as there were not enough pores with intermittency in the decane observations for a statistically meaningful analysis. For every pore, for all nitrogen experimental data, the pore occupancy of the surrounding pores is plotted against coordination number (the total number of directly connected pores) in Fig. 8(a). Figure 8(a) shows that for larger coordination numbers, it is more likely that the surrounding pores will be filled with nitrogen. There is a positive correlation between coordination number and pore size because larger pores are more likely to be directly connected to many other pores. Figure 8(a) simply reflects that the nonwetting phase occupies larger pores on average.

However, when considering only intermittent pores, there is an opposite trend in the occupancy of neighbors [Figs. 8(b) and $8(\mathrm{c})$ ]. Intermittent pores with higher coordination numbers are more likely to be surrounded by brine. This indicates that when the nonwetting phase occupies larger pores, intermittency will only occur when the surrounding pores are filled with brine.
There are two possible explanations for this correlation, which is strong enough to be seen over the relationship of coordination number and pore size. The first is that pores with high coordination numbers, with many surrounding pores filled with nonwetting phase, will not be intermittent because the nonwetting phase has connected pathways through which to flow. In contrast, if surrounding pores are filled with the wetting phase, then intermittent interface creation provides the only route for nonwetting phase flow. The second is that the filling mechanism is controlling the type of intermittency that can occur, and certain types of intermittent pathways are favorable. If the nonwetting phase invades a pore, then it will do so in a pistonlike fashion. The brine, as the wetting phase, can invade cooperatively if many of the surrounding pores are filled with brine; the more neighboring pores that are brine filled, the more favorable the displacement [21]. Dynamic imaging at higher temporal resolution would be required to prove these hypotheses and observe the potential for different types of intermittent pathway.

Figure 8(a) shows that the fraction of the surrounding pores filled with the intermittent phase decreases with coordination number. This implies that intermittent pathway flow is more important in poorly connected pores. The pores with lower coordination numbers make up the majority of the locations with intermittency displayed in Figs. 8(b) and 8(c).

We will now consider an intermittent pore with a coordination number of 2 to highlight the importance of intermittent 
Time averaged image of an intermittent pore with a coordination number of 2
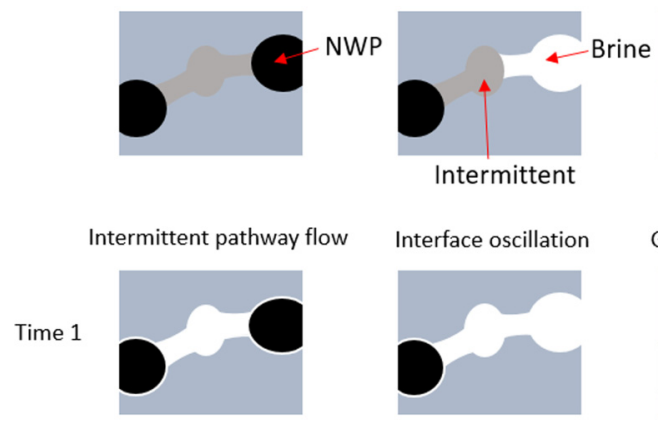

Interface oscillation

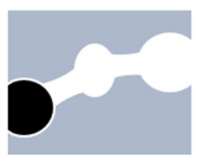

Ganglion movement

Time 2

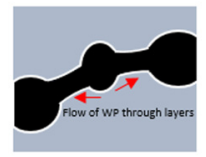

Time 3
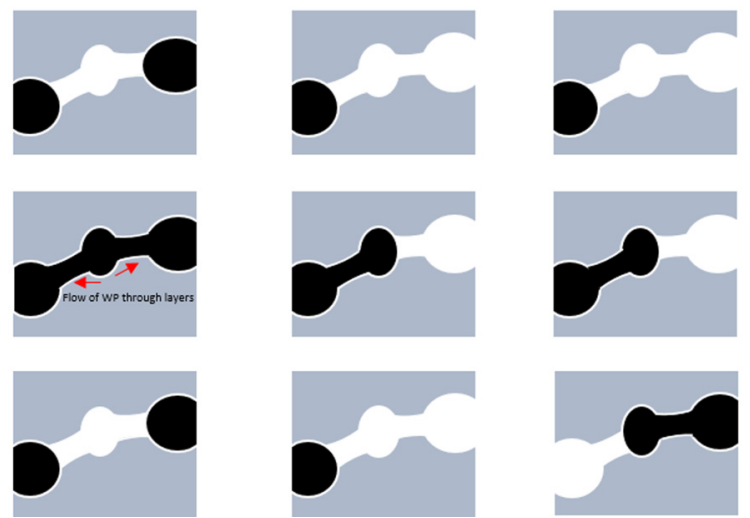

FIG. 9. Potential displacements for a pore, with a coordination number of 2, identified as intermittent in a scan. WP denotes the wetting phase (brine).

pathway flow relative to ganglion dynamics. See Fig. 8 for the data and Fig. 9 for a schematic representation. In this case, the fraction of the surrounding pores filled with nitrogen is approximately 0.7 , for both the low-Ca and high-Ca nitrogen observations and less than $10 \%$ of the surrounding pores are filled with brine [Figs. 8(b) and 8(c)]. This means that for the majority of cases, both the surrounding pores were filled with nitrogen, which results in the scenario shown in the left column of Fig. 9. In some cases, one of surrounding pores is filled with nitrogen and one other with brine (middle column in Fig. 9). This scenario may influence intermittent pathways in other areas of the pore space but does not contribute to flow. In some cases, an intermittent pore is surrounded by intermittent pores - this could be the scenario shown in the right column of Fig. 9, which would be the ganglion dynamics described in micromodel experiments by Avraam and Payatakes [12] where discrete blobs of the nonwetting phase advect through the pore space. However, manual inspection of the few regions with this configuration show that, for our observations, they are all larger-scale versions of the intermittency scenario shown in the left column of Fig. 9.

Overall, the analysis of these events reveals an important feature of intermittent flow: the nonwetting phase is not transported through the advection of small ganglia that are swept through the pore space, which is the traditional view of ganglion dynamics [12]. Rather, the fluid moves through the periodic opening of critical pathways that facilitate flow, akin to the operation of traffic lights [14]. Note that no cases were observed with intermittent pores surrounded by brine on both sides; this configuration would imply viscous ganglion dynamics, with ganglia on the size of a pore advecting through the void space [13].

\section{CONCLUSIONS}

In this work, intermittent pathway flow during steadystate two-phase flow was observed and quantified with timeaveraged laboratory micro-CT imagery for a carbonate rock. Intermittent pathway flow was observed as an exclusive mode of transport outside connected pathway flow in a regime dominated by capillary forces. It led to the interrupted transport of the nonwetting phase, which controls the connectivity of the nonwetting phase and its transport through the system. We evaluated the role of intermittency in the connectivity of the fluids and identified the role of pore structure in generating intermittent pathways.

Intermittent pathways are generated by the competition between the fluid phases, such that intermittency is observable in the intermediate sized pores, with the average size of intermittent events decreasing as the nonwetting is able to invade smaller pores. We also observed a dependency on the generation of intermittent pathways with the fractional flow. This relationship is observable in repeat experiments, and between subvolumes in a heterogeneous samples, although the exact location of intermittent events varies.

We discovered that intermittent pathways were favorable within the sample in the poorly connected pores or where the nonwetting phase itself was poorly connected. This means that the intermittent pathway in that location was crucial for transport of the nonwetting phase. This emphasizes that intermittent pathways cannot be ignored in calculating macroscopic flow properties as they occur in key locations contributing to the transport of the fluid. Note we have studied the impact of viscosity ratio on the degree of intermittency in our companion paper [19].

Future work could study a wider range of flow conditions, including mixed-wet media, where it has been suggested that intermittency is more important than in the water-wet rock studied here [16]. It would also be valuable to use fast synchrotron imaging to determine the frequency of intermittent displacement.

\section{ACKNOWLEDGMENTS}

Thanks to S. Berg, A. Scanziani, and G. Garfi for providing interesting debates and to Y. Gao, H. Menke, and K. Singh for their support during the experiments. C.S. is grateful for her funding through the President's PhD Scholarship, Imperial College London. The Shell Digital Rocks Programme, Imperial College London, is thanked for funding the experimental work. We are also grateful to all our colleagues within this programme for their useful discussions and support. T.B. acknowledges the Research Foundation-Flanders for his postdoctoral fellowship 12X0919N.
[1] R. Juanes, E. Spiteri, F. Orr, and M. J. Blunt, Water Resour. Res. 42, W12418 (2006).
[2] J. Niessner, S. Berg, and S. M. Hassanizadeh, Transp. Porous Media 88, 133 (2011). 
[3] M. Muskat, The Physical Principles of Oil Production (McGraw-Hill Book Company, New York, 1949).

[4] R. T. Armstrong, J. E. McClure, M. A. Berrill, M. Rücker, S. Schlüter, and S. Berg, Phys. Rev. E 94, 043113 (2016).

[5] M. J. Blunt, Multiphase Flow in Permeable Media: A Pore-Scale Perspective (Cambridge University Press, Cambridge, 2017).

[6] F. A. Dullien, Porous Media: Fluid Transport and Pore Structure (Academic Press, San Diego, CA, 1992).

[7] K. T. Tallakstad, H. A. Knudsen, T. Ramstad, G. Løvoll, K. J. Måløy, R. Toussaint, and E. G. Flekkøy, Phys. Rev. Lett. 102, 074502 (2009).

[8] K. T. Tallakstad, G. Løvoll, H. A. Knudsen, T. Ramstad, E. G. Flekkøy, and K. J. Måløy, Phys. Rev. E 80, 036308 (2009).

[9] H. A. Knudsen and A. Hansen, Europhys. Lett. 65, 200 (2004).

[10] L. A. Richards, Physics 1, 318 (1931).

[11] M. Leverett, Trans. AIME 142, 152 (1941).

[12] D. Avraam and A. Payatakes, J. Fluid Mech. 293, 207 (1995).

[13] S. S. Datta, T. Ramakrishnan, and D. A. Weitz, Phys. Fluids 26, 022002 (2014).

[14] C. A. Reynolds, H. Menke, M. Andrew, M. J. Blunt, and S. Krevor, Proc. Natl. Acad. Sci. USA 114, 8187 (2017).

[15] Y. Gao, Q. Lin, B. Bijeljic, and M. J. Blunt, Water Resour. Res. 53, 10274 (2017).

[16] M. Rücker, W.-B. Bartels, K. Singh, N. Brussee, A. Coorn, H. van der Linde, A. Bonnin, H. Ott, S. Hassanizadeh, M. J. Blunt, H. Mahani, A. Georgiadis, and S. Berg, Geophys. Res. Lett. 46, 3225 (2019).

[17] M. Rücker, S. Berg, R. Armstrong, A. Georgiadis, H. Ott, A. Schwing, R. Neiteler, N. Brussee, A. Makurat, L. Leu, M. Wolf, F. Khan, F. Enzmann, and M. Kersten, Geophys. Res. Lett. 42, 3888 (2015).
[18] Y. Gao, A. Q. Raeini, M. J. Blunt, and B. Bijeljic, Adv. Water Resour. 129, 56 (2019).

[19] C. Spurin, S. Krevor, T. Bultreys, B. Bijeljic, and M. Blunt (2019), https://doi.org/10.31223/osf.io/nm362.

[20] N. C. Wardlaw, J. Can. Pet. Technol. 21, 21 (1982).

[21] R. Lenormand, C. Zarcone, and A. Sarr, J. Fluid Mech. 135, 337 (1983).

[22] K. Singh, H. Menke, M. Andrew, Q. Lin, C. Rau, M. J. Blunt, and B. Bijeljic, Sci. Rep. 7, 5192 (2017).

[23] P. Lai, K. Moulton, and S. Krevor, Chem. Geol. 411, 260 (2015).

[24] M. Andrew, B. Bijeljic, and M. J. Blunt, Int. J. Greenhouse Gas Contr. 22, 1 (2014).

[25] R. C. Hardy, J. Res. Natl. Bur. Stand. 61, 433 (1958).

[26] A. Goebel and K. Lunkenheimer, Langmuir 13, 369 (1997).

[27] P. Soille, Morphological Image Analysis: Principles and Applications (Springer Science \& Business Media, New York, 2013), pp. 267-292.

[28] H. Dong and M. J. Blunt, Phys. Rev. E 80, 036307 (2009).

[29] A. Q. Raeini, B. Bijeljic, and M. J. Blunt, Phys. Rev. E 96, 013312 (2017).

[30] S. Berg, H. Ott, S. A. Klapp, A. Schwing, R. Neiteler, N. Brussee, A. Makurat, L. Leu, F. Enzmann, J.-O. Schwarz et al., Proc. Natl. Acad. Sci. USA 110, 3755 (2013).

[31] S. Zou, R. T. Armstrong, J.-Y. Arns, C. Arns, and F. Hussain, Water Resour. Res. 54, 3277 (2018).

[32] T. Bultreys, Q. Lin, Y. Gao, A. Q. Raeini, A. AlRatrout, B. Bijeljic, and M. J. Blunt, Phys. Rev. E 97, 053104 (2018).

[33] M. Erpelding, S. Sinha, K. T. Tallakstad, A. Hansen, E. G. Flekkøy, and K. J. Måløy, Phys. Rev. E 88, 053004 (2013). 